\title{
A DEEP 20 CM RADIO MOSAIC OF THE ESO KEY-PROJECT GALAXY REDSHIFT SURVEY
}

I. PRANDONI ${ }^{1}$, L. GREGORINI ${ }^{1}$, P. PARMA ${ }^{1}$, H.R. DE RUITER ${ }^{1}$, G. VETTOLANI ${ }^{1}$, M.H. WIERINGA ${ }^{2}$ AND R.D.EKERS ${ }^{2}$

${ }^{1}$ IRA - CNR, Via Gobetti 101, 40129 Bologna, Italy

${ }^{2}$ ATNF, P.O. Box 76, Epping NSW 2121, Australia

\section{Background: The Optical Sample}

In two strips of $22^{\circ} \times 1^{\circ}$ and $5^{\circ} \times 1^{\circ}$ near the SGP Vettolani et al. $(1993, I A U$ Symposium 161, "Astronomy from Wide Field Imaging", H.T. MacGillivray ed., Reidel, in press) have made a deep redshift survey as an ESO Key Project. All the galaxies down to $b_{J} \sim 19.4$ were observed with the OPTOPUS multi-fiber spectrograph on the $3.6 \mathrm{~m}$ telescope in La Silla, yielding 3348 redshifts. The survey has a typical depth of $z=0.1$. It fully samples the optical luminosity function down to $B=-15$ and various galaxy populations (e.g. normal galaxies, LSBDs and BCDs) are present. Interestingly, emission lines (OII, $\mathrm{H} \beta$, OIII) have been found in a large fraction of the galaxy spectra $(\sim 40 \%)$, suggesting strong evolution of the galaxy population in terms of enhanced star formation.

\section{ATCA Radio Observations: First Results}

We are using the ATCA at $20 \mathrm{~cm}$ to image the entire area of the optical survey (mosaic observing mode) with uniform sensitivity (336 pointings), needed for statistical studies. Since our optical sample is rather deep but narrow and 'normal' galaxies are tipically low-power radio sources, deep radio observations are needed. A $3 \sigma$ radio limit of $\sim 0.2 \mathrm{mJy}$ (allowed when sky positions are known) will enable us to detect $P<10^{21} \mathrm{~W} / \mathrm{Hz}$ for $z<0.1$. 


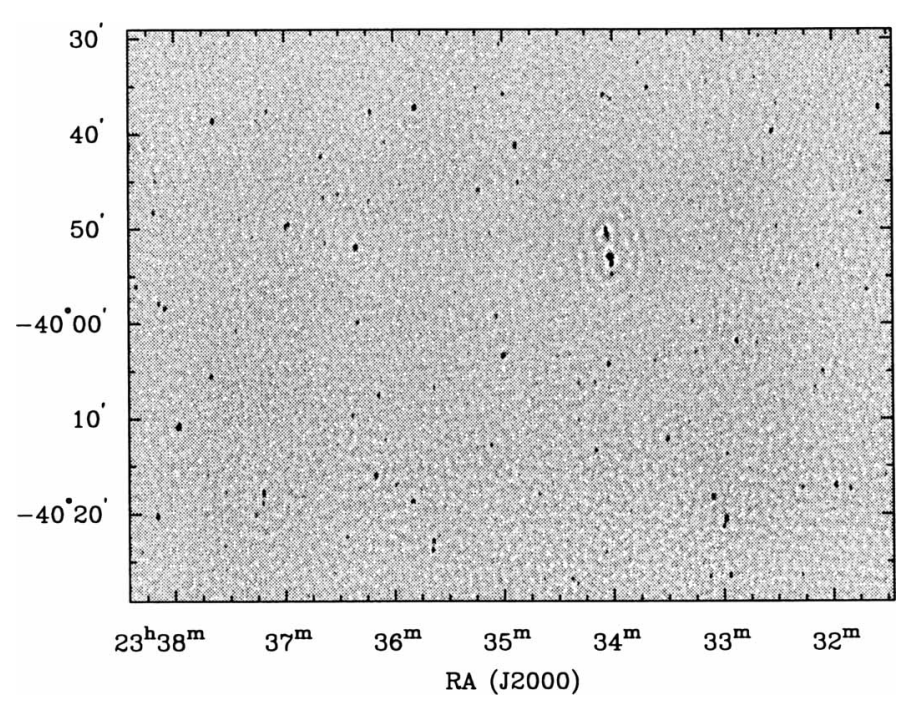

To reach this detection limit we need to observe 1.2 hours per field (with $2 \times 128 \mathrm{MHz}$ bandwidth). All 336 fields can thus be observed in 34 blocks of $12^{h}$. We have already observed $\sim 50 \%$ of the entire area and plan to complete the observations by the end of 1996 .

Data reduction is almost completed. We get cleaned mosaiced maps of 1.34 sq. degs. with spatial resolution $16^{\prime \prime} \times 8^{\prime \prime}$ (see Figure). The noise level is $\sim 70 \mu \mathrm{Jy}$ and is fairly uniform over the entire map. Dynamic range problems cause slightly higher levels of $\sim 80 \mu \mathrm{Jy}$ around strong sources $\left(S_{\text {peak }}>50 \mathrm{mJy}\right)$.

In a preliminary analysis of a 4 sq. degs. area we have searched for radio emission associated with the redshift survey galaxies. We used a $3 \sigma$ threshold of $0.24 \mathrm{mJy}$ (taking a conservative value of $80 \mu \mathrm{Jy}$ for the noise). We found 42 associations. A large fraction $(\sim 50 \%)$ of them are associated with galaxies showing one or more emission lines. We expect to detect $\sim 300$ galaxies in the entire area surveyed ( 27 sq. degs.). The numbers reported above allow us to believe that, when the survey will be completed, a very reliable statistical study of the existing correlations between optical (line activity, colors, morphologies, luminosities, etc.) and radio properties of the various populations represented in the optical sample will be possible.

In the same area we searched for all the radio sources above a $5 \sigma$ threshold of $0.4 \mathrm{mJy}$, getting 360 detections. $40 \%$ of them are sub-mJy objects. We expect a total number of $\sim 2500$ radio sources in the entire area surveyed. This new homogeneous and fairly deep sample of radio sources will allow better studies of e.g., the sub-mJy population, and be useful for the selection of appropriate subsamples aimed at different kinds of analysis. 